\title{
A Graphene-Edge Ferroelectric Molecular Switch
}

Caridad, José M.; Calogero, Gaetano; Pedrinazzi, Paolo; Santos, Jaime Eduardo Vieira da Silva Moutinho; Impellizzeri, Anthony; Gunst, Tue; Booth, Timothy J.; Sordan, Roman; Bøggild, Peter; Brandbyge, Mads

Published in:

Nano Letters

Link to article, DOI:

10.1021/acs.nanolett.8b00797

Publication date:

2018

Document Version

Publisher's PDF, also known as Version of record

Link back to DTU Orbit

Citation (APA):

Caridad, J. M., Calogero, G., Pedrinazzi, P., Santos, J. E. V. D. S. M., Impellizzeri, A., Gunst, T., Booth, T. J., Sordan, R., Bøggild, P., \& Brandbyge, M. (2018). A Graphene-Edge Ferroelectric Molecular Switch. Nano Letters, 18(8), 4675-4683. https://doi.org/10.1021/acs.nanolett.8b00797

\section{General rights}

Copyright and moral rights for the publications made accessible in the public portal are retained by the authors and/or other copyright owners and it is a condition of accessing publications that users recognise and abide by the legal requirements associated with these rights.

- Users may download and print one copy of any publication from the public portal for the purpose of private study or research.

- You may not further distribute the material or use it for any profit-making activity or commercial gain

- You may freely distribute the URL identifying the publication in the public portal 


\section{A Graphene-Edge Ferroelectric Molecular Switch}

\section{José M. Caridad, ${ }^{* \dagger}{ }^{\dagger}$ Gaetano Calogero, ${ }^{\dagger}$ Paolo Pedrinazzi, ${ }^{\dagger \dagger}{ }^{\dagger}$ Jaime E. Santos, ${ }^{\dagger, \S}$ Anthony Impellizzeri, ${ }^{\dagger, \|}$}

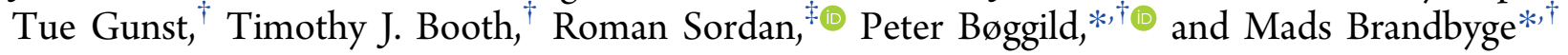

${ }^{\dagger}$ Center for Nanostructured Graphene (CNG), Department of Micro- and Nanotechnology, Technical University of Denmark, 2800 Kongens Lyngby, Denmark

${ }^{\ddagger}$ L-NESS Laboratory, Department of Physics, Politecnico di Milano, Via Anzani 42, 22100 Como, Italy

${ }^{\S}$ Centro de Física and Departamento de Física, Universidade do Minho, P-4710-057 Braga, Portugal

"Dipartimento di Fisica e Astronomia, Università di Catania, 64 Via Santa Sofia, 95123 Catania, Italy

\section{Supporting Information}

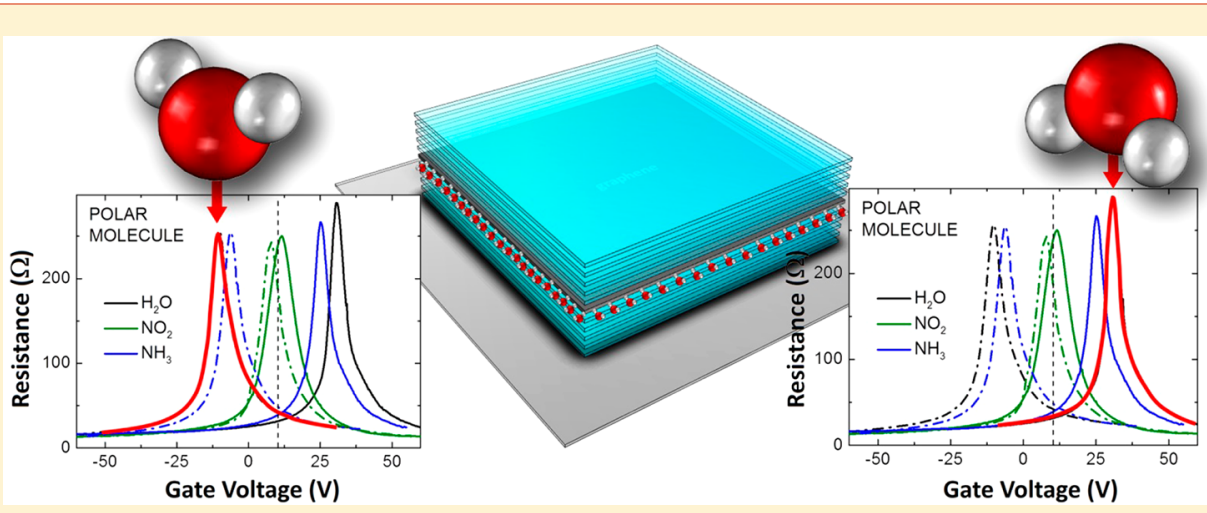

ABSTRACT: We show that polar molecules (water, ammonia, and nitrogen dioxide) adsorbed solely at the exposed edges of an encapsulated graphene sheet exhibit ferroelectricity, collectively orienting and switching reproducibly between two available states in response to an external electric field. This ferroelectric molecular switching introduces drastic modifications to the graphene bulk conductivity and produces a large and ambipolar charge bistability in micrometer-size graphene devices. This system comprises an experimental realization of envisioned memory capacitive ("memcapacitive") devices whose capacitance is a function of their charging history, here conceived via confined and correlated polar molecules at the one-dimensional edge of a two-dimensional crystal.

KEYWORDS: graphene edges, polar molecules, ferroelectricity, memcapacitor, hysteresis, molecular switch

M olecular switches comprise a new range of solid-state electronic systems able to reversibly shift molecules between two or more states. ${ }^{1,2}$ In recent years, several switches based on single molecules have been created on solid surfaces implementing simple functionalities such as transistors or tunable photoconductors. ${ }^{1,2}$ The development of more advanced architectures and electronic capabilities at a molecular level requires, however, the understanding and control of collective interactions between molecules composing these systems. ${ }^{3}$ Such a scenario may be created in molecules possessing long-range dipolar interactions confined to surfaces of reduced dimensionality, ${ }^{4}$ enabling the generation of correlated, ferroelectric systems with a switchable response. In the present work, we demonstrate that these novel functionalities can be achieved by confining polar molecules at the edge of a graphene sheet. Specifically, we show large, ambipolar charge bistabilities in the electrical characteristics of encapsulated, gated graphene devices with oxygenated edges exposed to gas environments containing different polar molecules. It is important to note that this ambipolar behavior does not occur in two distinct cases: (i) when the polar gas surrounding the oxygenated edges of graphene devices is replaced by a nonpolar gas, and (ii) when the edges of the encapsulated devices are fluorinated, regardless whether the environment contains polar gases or not. Both of these observations clearly point out that graphene edges and polar molecules are the key contributors to the observed phenomenon, indicating that polar molecules adsorbed at oxidized graphene edges become polarized, which we find affects the graphene bulk conductivity via capacitive coupling. In other words, the aforementioned observations cannot be ascribed to other device components such as the hexagonal boron nitride $(\mathrm{hBN})$ and $\mathrm{SiO}_{2}$ dielectrics or the metal contacts.

We provide a simplified atomistic model of the system based on Density Functional Theory (DFT) calculations, which is

Received: February 26, 2018

Revised: July 14, 2018

Published: July 20, 2018 
(a)
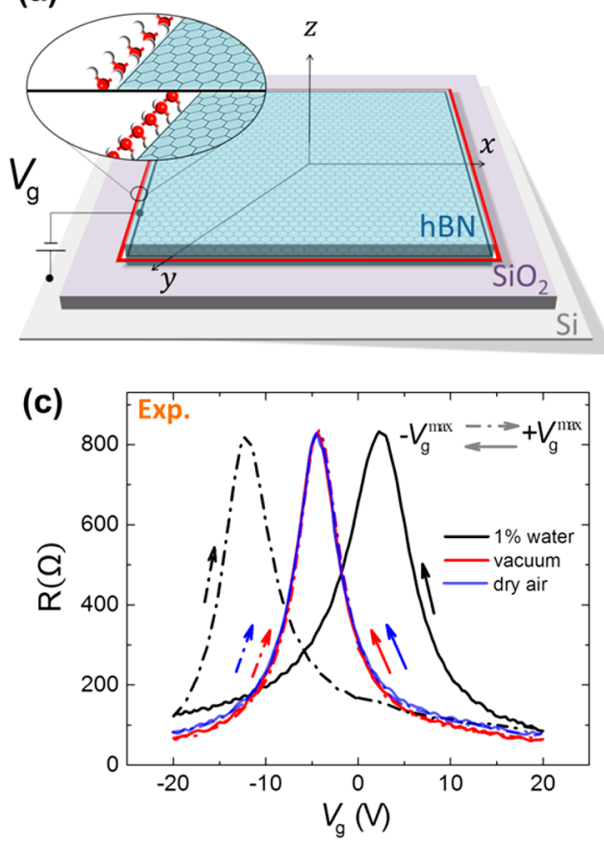
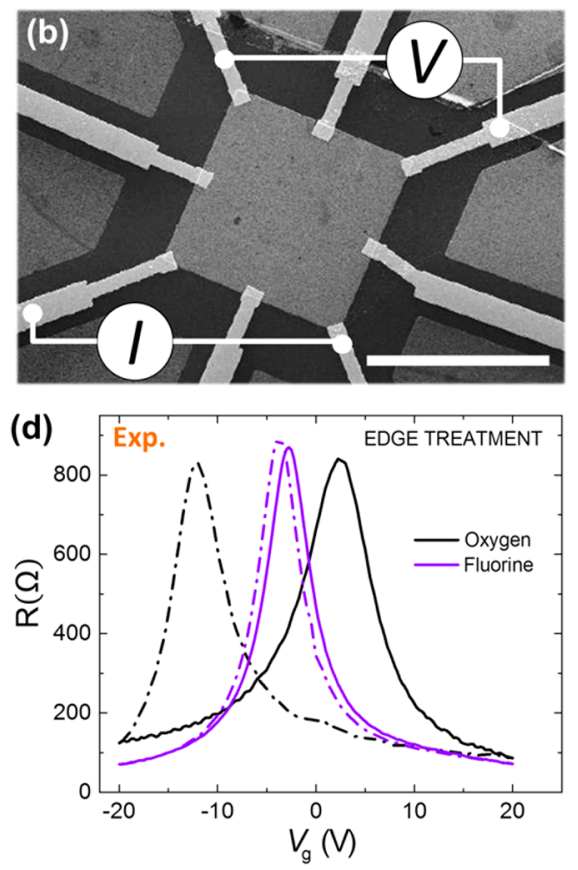

Figure 1. Polar molecules at graphene edges in micrometer-scaled gated devices. (a) Schematic of the squared graphene device encapsulated by hexagonal boron nitride (hBN) with water molecules at edges. Inset shows the two collectively aligned molecular configurations existing at graphene edges: above and below the graphene plane as further described below in Figure 3. (b) Scanning electron micrograph (SEM) of one of our devices. Scale bar is $5 \mu \mathrm{m}$. (c) Resistance $R=V / I$ measured across one of our devices (Device 1) with oxidized edges immersed in three different environments: vacuum (red), dry air (blue), and dry air with $1 \%$ water vapor (black). The resistance is measured following two sweep sequences: $+V_{\mathrm{g}}^{\max } \rightarrow-V_{\mathrm{g}}^{\max }$ (continuous line) and $-V_{\mathrm{g}}^{\max } \rightarrow+V_{\mathrm{g}}^{\max }$ (dashed-dotted lines). (d) Resistance $R=V / I$ measured across the same device (Device 1) after fluorine (violet) and oxygen (black) edge treatments in an environment composed of dry air with $1 \%$ water vapor.

able to capture the alignment of a single chain of polar molecules adsorbed at edges of gated graphene devices with oxygen termination. Combining these results with a phenomenological device model, we are able to reproduce our experiments in qualitative and quantitative terms. This overall ferroelectric system comprises an experimental instance of newly envisioned memory capacitive (memcapacitive) devices, ${ }^{5,6}$ here undertaken at a molecular level and with promising perspectives in terms of developing efficient circuitry for artificial neural computing. ${ }^{6,7}$

\section{RESULTS AND DISCUSSION}

The adsorption of molecular species on graphene has been extensively studied as an efficient approach to modify its electronic properties and can result in both reversible insertion of charge carriers in the monolayer ${ }^{8-10}$ and the modification of its electronic band structure. ${ }^{11,12}$ To date, the focus has largely been on adsorption on the graphene basal plane, assuming a negligible contribution from edge adsorbed species. The behavior of molecules adsorbed exclusively at the graphene edge remains relatively unexplored despite their critical role in determining the physical and chemical properties of this $2 \mathrm{D}$ crystal. ${ }^{13-16}$ Following published techniques for the exfoliation and van der Waals assembly of $2 \mathrm{D}$ materials, ${ }^{17-19}$ we have fabricated micrometer-size, encapsulated, graphene field-effect devices where only the graphene edges are exposed to the ambient environment in order to evaluate the adsorption of polar molecules at these locations (Figure 1a,b). We note that edges of these graphene devices are exposed to fluorine- and oxygen-based plasma treatments during and after fabrication, which induces their fluorination or oxidation, respectively (details in Methods).
Figure 1c shows the measured resistance $R=V / I$ of one of our devices (Device 1) treated with oxygen plasma as a function of the gate-voltage $V_{\mathrm{g}}$ under three different environmental conditions: vacuum ( $<10^{-3}$ mbar, red), dry air (blue), and dry air mixed with $1 \%$ water vapor (black). In each of these cases, we have performed two gate-voltage sweeps in opposite directions, either starting from a positive or negative maximum gate-voltage $V_{\mathrm{g}}^{\max }$. Results for each of the three environmental conditions are shown with the negative sweep direction $+V_{\mathrm{g}}^{\max } \rightarrow-V_{\mathrm{g}}^{\max }$ as continuous lines and the positive sweep direction $-V_{\mathrm{g}}^{\max } \rightarrow+V_{\mathrm{g}}^{\max }$ as dashed-dotted lines. For both the vacuum and dry air curves, the resistance $R\left(V_{\mathrm{g}}\right)$ is independent of the sweep direction, with a charge neutrality point $V_{\mathrm{CNP}}$ located at $-4 \mathrm{~V}\left(V_{\mathrm{CNP}}^{0}\right)$ due to residual dopants near the graphene layer. ${ }^{17-19}$

Strikingly, the charge neutrality point in $R\left(V_{\mathrm{g}}\right)$ under $1 \%$ water vapor is shifted symmetrically around $V_{\mathrm{CNP}}^{0}$ by $\Delta V_{\mathrm{CNP}} \approx$ $+7 \mathrm{~V}$ or $-7 \mathrm{~V}$ when starting the gate-sweep from $+V_{\mathrm{g}}^{\max }$ or $-V_{\mathrm{g}}^{\max }$, respectively. In other words, our graphene device with oxygen-plasma-treated edges exhibits large, symmetric, and switchable episodes of homogeneous $p$ - or $n$ - doping depending on the respective starting point of the sweep sequence. This hysteretic doping behavior is almost completely quenched $\left(\Delta V_{\mathrm{CNP}} \approx 0.5 \mathrm{~V}\right)$ in the same encapsulated device after the fluorine-based plasma treatment, as shown in Figure 1d. The residual $\Delta V_{\mathrm{CNP}}=0.5 \mathrm{~V}$ can be accounted for by imperfect fluorine functionalization of the graphene edges. ${ }^{20}$

The observation of symmetrical (gate voltage history dependent) doping in devices with oxygen-treated graphene edges in the presence of water vapor indicates that interactions between water molecules and the oxidized graphene edge are responsible for the observed charge bistability, while other 
parts of the device, such as the $\mathrm{hBN}$ and $\mathrm{SiO}_{2}$ dielectrics or the metal contacts, are not. Specifically, this symmetric doping behavior show that water molecules adsorbed at graphene edges become somehow polarized in two macroscopic, preferential configurations (schematic in Figure 1a, inset, and discussion below) when conducting gate sweeps in our devices. Additional reasoning regarding why this charge bistability phenomenon cannot be accounted for by commonly reported hysteretic effects in literature are described in the Supporting Information, Note 1. We have confirmed the appearance of this phenomenon in multiple encapsulated devices with oxidized graphene edges and water molecules (Figure 2 and Supporting Information, Note 1). In addition, we have carried out three additional experiments in order to clarify the nature of this effect.

First, we show that $\Delta V_{\mathrm{CNP}}$ increases by increasing the magnitude of the maximum gate voltage $V_{\mathrm{g}}^{\max }$ in the sweep (Figure 2a). This observation shows that the applied gate voltage (or correspondingly, the electric field existing at graphene edges $E_{\text {loc }}{ }^{21,22}$ see Supporting Information, Note 2) impacts the actual microscopic structure and alignment of the adsorbed water molecules at graphene edges, suggesting that a larger applied electric field results in a higher degree of molecular polarization of water at the edges.

Second, we observe that the initial application of a gate voltage (electric field) from $V_{\mathrm{g}}=0 \mathrm{~V}$ does in effect polarize and align water molecules adsorbed at edges, as found from consecutive gate voltage sweeps $0 \rightarrow+V_{\mathrm{g}}^{\max } \rightarrow 0$. Figure $2 \mathrm{~b}$ shows that at $t=0$, when sweeping $V_{\mathrm{g}}$ from $0 \rightarrow+V_{\mathrm{g}}^{\max }$, the $V_{\mathrm{CNP}}$ of the device is close to $V_{\mathrm{g}}=0 \mathrm{~V}$ (corresponding to the value measured in vacuum $V_{\mathrm{CNP}}^{0}$ for this device). When sweeping from $+V_{\mathrm{g}}^{\max } \rightarrow 0$ the $V_{\mathrm{CNP}}$ of the device has shifted substantially to $\approx 20 \mathrm{~V}\left(V_{\mathrm{CNP}}^{*}\right)$. These trends are consistent with molecules becoming polarized at graphene edges. After waiting $30 \mathrm{~s}$ at $V_{\mathrm{g}}=0 \mathrm{~V}$, we repeat the sweep $0 \rightarrow V_{\mathrm{g}}^{\max }$. The $V_{\mathrm{CNP}}$ of the device is seen to remain at $V_{\mathrm{g}} \approx 20 \mathrm{~V}\left(V_{\mathrm{CNP}}^{*}\right)$ instead of returning to $V_{\mathrm{CNP}}^{0}$, clearly indicating a stable polarization of the water molecules at the graphene edge.

Third, we underpin that this phenomenon also occurs for other polar molecules. To test this, we consecutively expose one device to three individual environments composed of one of the following polar substances: water vapor $\mathrm{H}_{2} \mathrm{O}$, ammonia $\mathrm{NH}_{3}$ or nitrogen dioxide $\mathrm{NO}_{2}$, at a concentration of $1 \%$ in dry air. Prior to and between measurements in each of the three environments, the chamber was evacuated to $<10^{-3}$ mbar to avoid cross-contamination, and the return of the $V_{\mathrm{CNP}}$ to the original position of $V_{\mathrm{CNP}}^{0}$ was confirmed. Figure $2 \mathrm{c}$ shows that exposure to these three different polar molecules all exhibit the same previously observed episodes of symmetric and ambipolar doping, although to different degrees. This strongly indicates that the mechanism responsible of this phenomenon is general for polar molecules and effectively rules out exclusive interactions occurring within water molecules, ${ }^{23}$ oxygen-water or even carbon-water interactions, as causing this effect. More importantly, this observation clearly implies Coulomb interactions between polar molecules and between polar molecules and the (chemistry-dependent) graphene edge potential $^{13}$ as the relevant microscopic causes of the observed two-state macroscopic polarization of polar molecules adsorbed at the oxidized edges of encapsulated graphene devices.

We note that ferroelectricity is predicted ${ }^{24,25}$ and claimed ${ }^{26}$ to occur in confined systems with ordered polar molecules as a
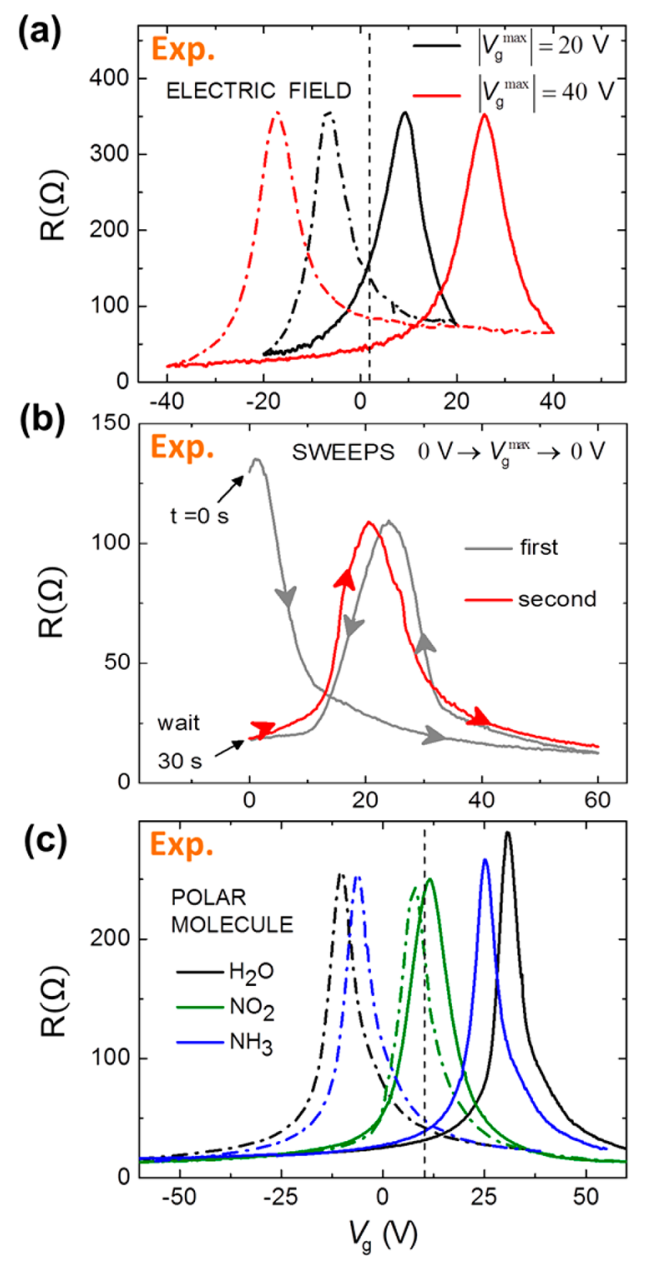

Figure 2. Electrical measurements of micrometer-size gated graphene devices in different conditions. (a) $R$ measured across one of our devices (Device 2) with oxygen edge-treatment immersed in dry air with $1 \%$ water vapor following two sweep sequences: $+V_{\mathrm{g}}^{\max } \rightarrow-V_{\mathrm{g}}^{\max }$ (continuous line) and $-V_{\mathrm{g}}^{\max } \rightarrow+V_{\mathrm{g}}^{\max }$ (dashed-dotted lines). Here we took two values for $\left|V_{\mathrm{g}}^{\mathrm{max}}\right|: 20 \mathrm{~V}$ (black) and $40 \mathrm{~V}$ (red). (b) Change of charge neutrality point in one of our devices (Device 3 ) with oxygen edge-treatment immersed in dry air with $1 \%$ water vapor when sweeping $V_{\mathrm{g}}$ following the sequence $0 \rightarrow V_{\mathrm{g}}^{\max } \rightarrow 0$ two consecutive times. During the first sweep, the charge neutrality point changed from $V_{\mathrm{CNP}}^{0} \approx 0.5 \mathrm{~V}$ to $V_{\mathrm{CNP}}^{*} \approx 20 \mathrm{~V}$, indicating that molecules became polarized. After waiting $30 \mathrm{~s}$ at $V_{\mathrm{g}}=0 \mathrm{~V}$, a second sweep $0 \rightarrow V_{\mathrm{g}}^{\max }$ was undertaken. The charge neutrality point clearly remained at the position $V_{\mathrm{CNP}}^{*}$; that is, molecules stay polarized (albeit a small shift due to depolarization effects occurring at room temperature). (c) $R$ measured across one of our devices (Device 4) with oxygen edgetreatment immersed in dry air with $1 \%$ water vapor (black), dry air with $1 \%$ nitrogen dioxide (green), and dry air with $1 \%$ ammonia (blue). Vertical dashed lines in panels (a) and (c) indicate the position of the charge neutrality point when devices are measured in vacuum.

result of enhanced dipolar interactions appearing in systems with reduced dimensionality. ${ }^{25,26}$ Figure $2 \mathrm{c}$ shows how $\Delta V_{\mathrm{CNP}}$ progressively increases for the $\mathrm{NO}_{2}, \mathrm{NH}_{3}$, and $\mathrm{H}_{2} \mathrm{O}$ cases, respectively-a behavior that is readily explained as a direct consequence of the different dipole moment $\mu_{\mathrm{d}}$ of these molecules: $\mu_{\mathrm{d}}^{\mathrm{NO}_{2}}(0.4 \mathrm{D})<\mu_{\mathrm{d}}^{\mathrm{NH}_{3}}(1.74 \mathrm{D})<\mu_{\mathrm{d}}^{\mathrm{H}_{2} \mathrm{O}}(1.85 \mathrm{D})$ (see eq 1 and the analytical model proposed below).

The experimentally observed charge bistability is seen only for oxygen-plasma-treated graphene edges exposed to polar 

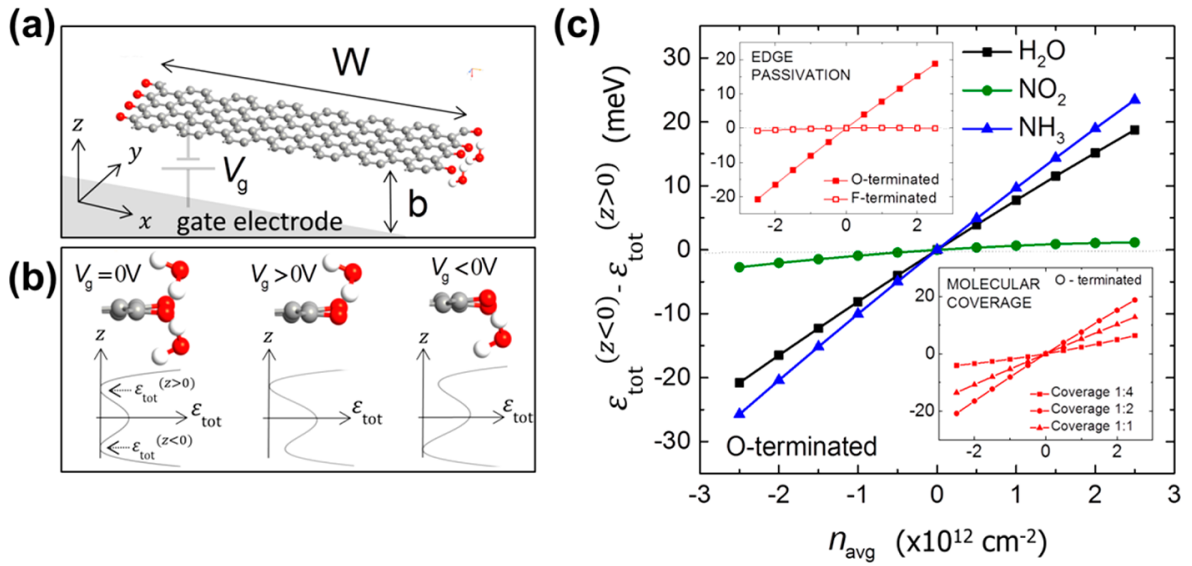

Figure 3. Atomistic DFT calculations of gated nanoribbons with adsorption of molecules at graphene edges. (a) Simulated graphene ribbon (periodic in the $y$ direction) of width $W(3,13$, and $23 \mathrm{~nm}$ ) with a gate electrode underneath at a distance $b$. (b) Two stable configurations ("above" and "below" the graphene plane w.r.t. the gate) of water molecules at graphene edges (passivated with oxygen) and preferential placement depending on $V_{\mathrm{g}}$. Graphs below show an schematic of the total energy $\varepsilon_{\text {tot }}$ (represented as a double well with two minimum positions below $\varepsilon_{\text {tot }}^{(z<0)}$ and above $\varepsilon_{\text {tot }}^{(z>0)^{g}}$ the graphene plane w.r.t. the gate electrode) and its evolution with $V_{\mathrm{g}}$. (c) Total energy difference between the two minimums (stable molecular position), at several averaged carrier densities $n_{\text {avg }}$ for three different types of polar molecule: water $\left(\mathrm{H}_{2} \mathrm{O}\right)$, nitrogen dioxide $\left(\mathrm{NO}_{2}\right)$, and ammonia $\left(\mathrm{NH}_{3}\right) \cdot \varepsilon_{\text {tot }}^{(z<0)}-\varepsilon_{\text {tot }}^{(z>0)}$ shows additional dependencies on edge termination (upper inset, simulation done with $\mathrm{H}_{2} \mathrm{O}$ ), type of polar molecule at the edge, and edge coverage (lower inset, simulation done with $\mathrm{H}_{2} \mathrm{O}$ ).

species. For ferroelectric ordering of polar edge molecules to appear and cause a measurable change in the electrical response of encapsulated graphene devices, we require two conditions to be met: (i) a stable (but not necessarily complete) alignment of polar molecules as obtained upon application of an external electric field to the device, and (ii) interactions between the polar molecular ensemble and the chemistry-dependent graphene edge as well as intermolecular Coulomb interactions.

Alignment of Polar Molecules at Graphene Edges. We use a simple atomistic model in order to understand the binding energy $\varepsilon_{\mathrm{b}}$ of polar molecules adsorbed at the edges of a finite-size graphene sheet for different edge passivation using Density Functional Theory (DFT) with an incorporated gate field (Methods). This model, Figure 3a, includes the key elements of the experimental system, comprising the gating of a finite-size graphene sheet modeled here as a nanoribbon with oxidized or fluorinated edges and the presence of polar molecules in the proximity of these edges (see Methods). Complete details of the equilibrium positions and the calculation of the binding energies of all combinations of polar molecules $\left(\mathrm{H}_{2} \mathrm{O}, \mathrm{NH}_{3}, \mathrm{NO}_{2}\right)$ and graphene terminations (oxygen, fluorine) considered in our experiments can be found in Supporting Information, Notes 3 and 4. Numerical values for the binding energies of all the tested cases are presented in Table 1 . In general, values of $\varepsilon_{\mathrm{b}}$ between 0.5 and $1 \mathrm{eV}$ are obtained for all three polar molecules adsorbed at oxidized graphene edges, close to those reported in literature. ${ }^{14,15}$ Instead $\varepsilon_{\mathrm{b}}$ is more than 3 times smaller for the calculated case of $\mathrm{H}_{2} \mathrm{O}$ adsorbed at fluorinated graphene edges. This behavior

Table 1. Calculated Binding Energies of Different Molecules Adsorbed at Graphene Edges with Different Terminations

\begin{tabular}{clc} 
edge termination & \multicolumn{1}{c}{ polar molecule } & $\varepsilon_{\mathrm{b}}(\mathrm{eV})$ \\
oxygen & water $\left(\mathrm{H}_{2} \mathrm{O}\right)$ & 0.508 \\
oxygen & nitrogen dioxide $\left(\mathrm{NO}_{2}\right)$ & 0.937 \\
oxygen & ammonia $\left(\mathrm{NH}_{3}\right)$ & 0.534 \\
fluorine & water $\left(\mathrm{H}_{2} \mathrm{O}\right)$ & 0.159
\end{tabular}

agrees with the fact that $\mathrm{C}-\mathrm{O}$ bonds at the edges enhance the adsorption strength of polar molecules at those positions. ${ }^{14,15}$

Figure $3 \mathrm{~b}$ shows that at $V_{\mathrm{g}}=0 \mathrm{~V}$, after relaxing the structure, individual polar molecules are randomly trapped in one of only two allowed equilibrium positions. These positions are symmetrically oriented with respect to the graphene basal plane, a behavior that has already been reported in previous studies. ${ }^{15}$ Importantly, this equally probable trapping is broken for devices with oxygenated edges when $V_{\mathrm{g}} \neq 0 \mathrm{~V}$, i.e., in the presence of the local electric field $E_{\text {loc }}$ existing at the edge of finite-size graphene devices. ${ }^{21,22} \mathrm{We}$ demonstrate this by calculating (Figure 3c) the total energy difference of the system $\left(\varepsilon_{\text {tot }}^{(z<0)}-\varepsilon_{\text {tot }}^{(z>0)}\right)$ when the polar molecules are placed below $\varepsilon_{\text {tot }}^{(z<0)}$ and above $\varepsilon_{\text {tot }}^{(z<0)}$ the graphene plane $(z=0)$, for different averaged carrier densities in the device $n_{\text {avg }}$ (or equivalently $V_{\mathrm{g}}$ or $\left.E_{\mathrm{loc}}\right)$. The energy difference $\varepsilon_{\text {tot }}^{(z<0)}-\varepsilon_{\text {tot }}^{(z<0)}$ increases linearly with $n_{\text {avg }}\left(\right.$ or $\left.V_{\mathrm{g}}, E_{\mathrm{loc}}\right)$, crosses the origin, and changes sign with $n_{\text {avg }}$ (or $V_{g}, E_{\text {loc }}$ ). First, this behavior indicates that one of the two available configurations is favored for $V_{\mathrm{g}} \neq 0 \mathrm{~V}$ for oxygenated graphene edges. Second, a larger fraction of the population of polar molecules are positioned in the more favorable configuration for larger $n_{\text {avg }}$, with the final occupation depending ultimately on the maximum value of carrier density reached $n_{\mathrm{avg}}^{\max }$ (equivalently $E_{\mathrm{loc}}^{\max }$ or $V_{\mathrm{g}}^{\max }$ ). Finally, the more favorable position for an individual molecule flips upon changing the sign of $n_{\text {avg }}$ i.e., the system is ambipolar. These behaviors are qualitatively identical irrespective of the studied polar species, but quantitative differences in $\varepsilon_{\text {tot }}^{(z<0)}-$ $\varepsilon_{\text {tot }}^{(z<0)}$ between different molecules at any fixed $n_{\text {avg }}$ are observed (i.e., different slopes) and are present even for a full edge coverage (Figure 3c, lower inset).

These simulations agree with our experimental trends. In particular, the favoring of one of two available positions for the polar molecules at the graphene edge when $V_{\mathrm{g}} \neq 0 \mathrm{~V}$ is consistent with the observed bistable macroscopic charge states in our gated device. Furthermore, the population of these two molecular states in the system (i.e., molecular alignment) is indeed regulated by the external field $E_{\text {loc }}$ and depends quantitatively on the actual dipole moment of the 
polar molecule, as observed in experiments (Figure 2a,c). Moreover, the preferential trapping in any of the two calculated possible configurations observed in devices with oxygenated edges does not exist in the case of fluorinated edges for any $V_{\mathrm{g}}$. Figure $3 \mathrm{c}$ (upper inset) clearly shows that the energy difference $\varepsilon_{\text {tot }}^{(z<0)}-\varepsilon_{\text {tot }}^{(z<0)}$ does not change with $n_{\text {avg }}\left(V_{\mathrm{g}}\right.$ or $\left.E_{\text {loc }}\right)$ in devices with fluorine passivated edges. This indicates that in this case, the population of the two states is not controlled by an external field, in perfect agreement with the quenched charge bistability observed in our encapsulated graphene devices after a fluorine-based plasma treatment (Figure 1d).

Finally, we note that the DFT results are robust upon variations of the choice of functional (Methods), the width of our simulated graphene nanoribbon (Methods) and the edge chirality of the device: zigzag or armchair (Supporting Information, Note 5). The latter fact provides a compelling reason for the charge bistability phenomenon being present in our experimental graphene devices, having edge roughness $\sim 1$ $\mathrm{nm}^{22}$ and thus likely composed of a mixture of both edge chiralities. We also note that despite not accounting for temperature and pressure in our DFT calculations, we predict complete (1:1) edge coverage of polar molecules in our devices under the given experimental conditions by statistical mechanics calculations (Supporting Information, Note 6). Such calculations explicitly account for the effect of molecular mobility (rotation, vibration) and the possible adsorption and desorption of polar species from graphene edges at the given temperature and pressure of the experiments.

Capacitive Coupling of Aligned Polar Molecules to Graphene Device Characteristics. Collectively oriented and correlated polar molecules favoring either of the two observed states at graphene edges would generate a net dipole moment with a component perpendicular to the graphene plane $p_{z}$. We demonstrate below that $p_{z}$ has a measurable effect on the electrical characteristics $R\left(V_{\mathrm{g}} p_{z}\right)$ of a micrometer-size graphene device via capacitive coupling. Up to a first approximation, $p_{z}$ will depend on $^{27}$

$$
p_{z}=\mu_{\mathrm{d}} N \eta
$$

where $\mu_{\mathrm{d}}$ is the dipole moment of a single polar molecule, $N$ the total number of adsorbed polar molecules at the edges (corresponding in our case to the total number of available binding sites, see above and Supporting Information, Note 6), and $\eta=\left(N^{(z>0)}-N^{(z<0)}\right) N^{-1}$, the relative difference in the number of molecules above $\left(N^{(z>0)}\right)$ and below $\left(N^{(z<0)}\right)$ the graphene plane with respect to the gate electrode. Considering $\mu_{\mathrm{d}}$ and $N$ as fixed parameters for a given system, $-1<\eta<1$ determines its current state and consequent behavior.

The resistance $R$ of a micrometer-size, gated graphene transistor is inversely proportional to the total charge $Q$ that exists in the monolayer: ${ }^{28}$

$$
R \propto \frac{1}{\sqrt{Q^{2}+Q_{\mathrm{res}}{ }^{2}}}
$$

where $Q_{\text {res }}$ is a residual charge due to unwanted doping and/or thermally activated carriers. ${ }^{28}$ In the present case, $Q$ will depend on both the back gate-voltage $V_{\mathrm{g}}$ and the net dipole moment $p_{z}$ along the edge of graphene $Q\left(V_{g}, p_{z}\right)$ and can be calculated electrostatically from the two contributions to the total surface charge density $\sigma=\sigma_{\mathrm{B}}+\sigma_{\mathrm{C}}$. Here, $\sigma_{\mathrm{B}}$ represents the bulk contribution (solely) due to the direct gate-induced carrier injection in the system. Meanwhile, $\sigma_{\mathrm{C}}$ is the additional contribution due to the aligned polar molecules, which have a notable effect close to the edges of the device. The detailed description of this electrostatic model appears in the Supporting Information, Note 7 . We note that $\sigma_{\mathrm{C}}$ and $\eta$ are the only free parameters of this electrostatic model and can be extracted from the experimental data. Moreover, $\sigma_{\mathrm{C}}$ depends on $\eta$, as we demonstrate below by a mean-field calculation reflecting the molecular switching mechanism. Therefore, $\eta$ is the only independent parameter of the proposed phenomenological model.

Figure $4 \mathrm{a}$ shows the calculated resistance of a micrometerscale graphene device for three cases: absence of polar molecules $\left(p_{z}=0\right)$ and full edge occupancy of polar molecules all oriented in one of the two possible orientations, (e.g., at $\left|\eta^{\max }\right|=1$ and $\left.\left|p_{z}\right|=\mu_{\mathrm{d}} N\right)$. The experimentally observed ambipolar resistance $R\left(V_{\mathrm{g}}, p_{z}\right)$ is clearly reproduced by the calculations.

Specifically, we took $\mu_{\mathrm{d}}=1.85 \mathrm{D}$ as the dipole moment of water while $N$ is estimated for full coverage as $N=4 L / a_{\mathrm{b}}$, where $4 L$ is the perimeter of our device and $a_{\mathrm{b}}$ is the mean distance between binding sites at the graphene edge (given by the second nearest neighbor distance in a graphene lattice, $\sim 0.25 \mathrm{~nm}$ ). With these assumptions, the separation $\Delta V_{\mathrm{CNP}}$ between resistance curves agrees quantitatively with our experiments (Figure 1c) for $\sigma_{\mathrm{C}}^{\max }=0.08 \mathrm{C} / \mathrm{m}^{2}$ in this case. The evolution of $\sigma_{\mathrm{C}}$ (or equivalently $\eta$ ) with respect to $V_{\mathrm{g}}$ therefore describes the ferroelectricity of the system.

Molecular Switching Mechanism: Mean-Field Model. We propose a ferroelectric model of molecular switching to simultaneously estimate the fraction $\eta\left(V_{\mathrm{g}}, T\right)$ of polarized molecules at a given $V_{\mathrm{g}}$ and temperature $T$, or equivalently the charge distribution induced in our graphene device due to the presence of polar molecules $\sigma_{\mathrm{C}}\left(V_{\mathrm{g}}, T\right)$. The ferroelectric switching model for an ensemble of molecules is based on the observed charge bistability in our experiments and is inspired by the mean-field Curie-Weiss model of magnetism. Using Boltzmann statistics, at a temperature $T$ and for a given back-gate generated local electric field $E_{\text {loc }}$ at the edge of the device, the probability of molecules being either above or below the graphene plane is

$$
N^{(z>0)}=\mathrm{e}^{\Delta \varepsilon / k_{\mathrm{B}} T}, \quad N^{(z<0)}=\mathrm{e}^{-\Delta \varepsilon / k_{\mathrm{B}} T}
$$

where the energy $\Delta \varepsilon=E_{\text {eff }} \mu_{\mathrm{d}} \equiv\left(K \eta+b_{i}\right) k_{\mathrm{B}} T$ represents the energy to flip a single dipole $\mu_{\mathrm{d}}$ in the effective electric field $E_{\text {eff }}$ existing at the edge for $V_{\mathrm{g}} \neq 0$ and finite molecular polarization. The term $b_{i}=E_{\mathrm{loc}} \mu_{\mathrm{d}} / k_{B} T$ contains all the interactions to which a single dipole is subjected given an electric field $E_{\text {loc }}$ which is the electric field for $\eta=0$ in the device. Meanwhile, $K \eta=\Delta E_{\text {eff }} \mu_{\mathrm{d}} / k_{\mathrm{B}} T$ describes the response to the local field at the edge $\Delta E_{\text {eff }}=E_{\text {eff }}-E_{\text {loc }}$ when $\eta \neq 0$. This effective interaction parameter $K$ depicts the charge induction in the graphene device due to the molecular polarization at edges, which, in turn, change the local field at the edge and provide a feedback mechanism between local field and polarization.

Given $\eta=\left(N^{(z>0)}-N^{(z<0)}\right) N^{-1}$, our problem is reduced to solving numerically the transcendental equation $\eta=\tanh (K \eta+$ $b_{i}$ ) (Figure $\left.4 \mathrm{~b}\right)$. Using eqs 1 and $2, R\left(V_{\mathrm{g}}, p_{z}\right)$ can be calculated; the results are shown in Figure $4 c$.

Importantly, in this model $K$ is the only free parameter (since $\Delta E_{\text {eff }}$ is related to $\sigma_{\mathrm{C}}(r)$, Supporting Information, Note $8)$ and takes into account the actual microscopic arrangement 

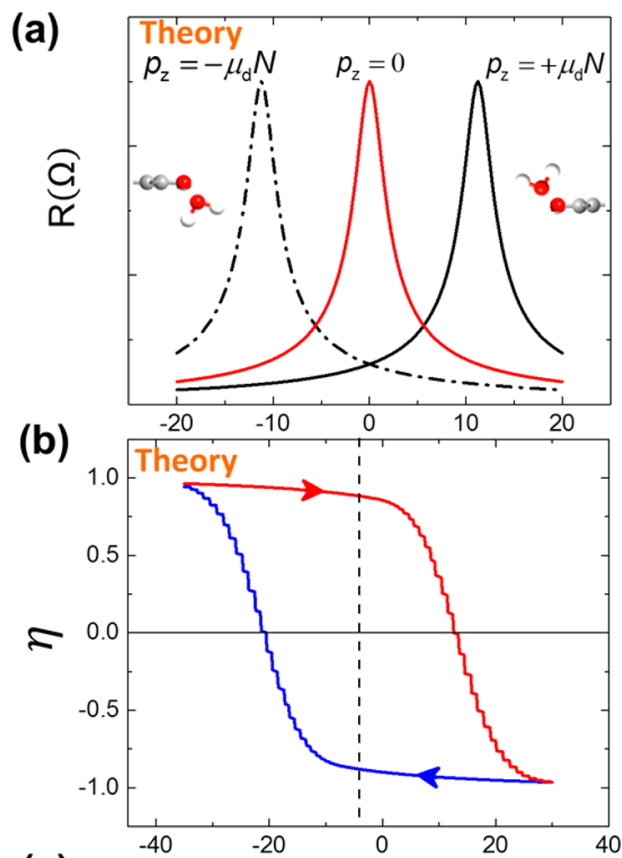

(c)

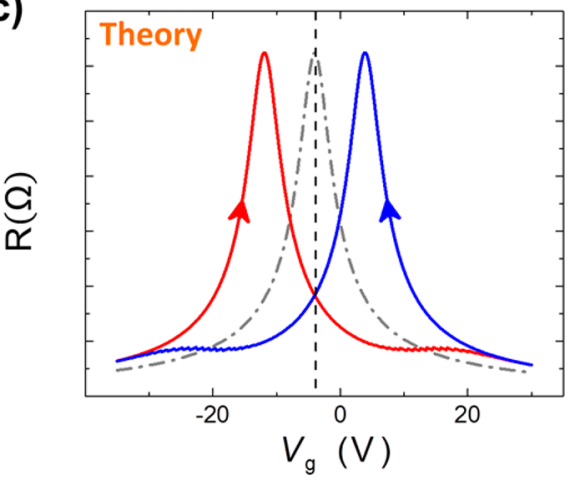

Figure 4. Ferroelectricity of polar molecules at the edges of micrometer-size graphene devices. (a) Electrostatic model, showing the calculated resistance in the device for different net dipole moments along the graphene edge: molecules above the graphene plane $\left(p_{z}=+\mu_{\mathrm{d}} N\right)$, molecules below the graphene plane $\left(p_{z}=-\mu_{\mathrm{d}} N\right)$, and absence of molecules $\left(p_{z}=0\right)$. A net dipole $p_{z}$ along a graphene edge introduces a considerable ambipolar doping (shifts in $V_{\mathrm{CNP}}$ ) in a micrometer size graphene device, symmetric for the two opposite molecular orientations. (b) Calculated transcendental equation $\eta=$ $\tanh \left(K \eta+b_{i}\right)$, showing hysteretic behavior for cyclic gate-sweeps. (c) Calculated resistance $R\left(V_{\mathrm{g},} p_{z}\right)$, including the $\eta$ obtained in panel (b), reflecting the observed hysteretic behavior in our experiments (Figure 1c). In our model of panels (b) and (c), we account for the $V_{\mathrm{CNP}}^{0}$ seen in our experimental device (Device 1, Figure 1) as indicated by vertical dashed lines.

of the polar molecules, including, for instance, situations where more than one row of molecules are present at the graphene edge. Given the experimentally observed $\left|\Delta V_{\mathrm{CNP}}\right|$, this meanfield model enables independent determination of $K$, the maximum polarization achieved in the system $\eta^{\max }$, and the surface charge density $\sigma_{\mathrm{C}}^{\max }$ of the corresponding device (see Supporting Information, Note 8). For the specific case of our experimental device in Figure 1c with $\left|\Delta V_{\mathrm{CNP}}\right| \approx 7 \mathrm{~V}$, these values are $\eta^{\max } \approx 0.84, K \approx 1.4$, and $\sigma_{\mathrm{C}}^{\max } \approx 0.03 \mathrm{C} / \mathrm{m}^{2}$. The values obtained for $\eta^{\max }$ and $\sigma_{\mathrm{C}}$ are consistent with those previously assumed $\left(\eta^{\max }=1\right)$ and estimated $\left(\sigma_{\mathrm{C}}^{\max } \approx 0.08 \mathrm{C}\right)$ $\mathrm{m}^{2}$ ) above from electrostatic calculations.
Therefore, $K$ is the key parameter describing the observed ferroelectric behavior of polar molecules at graphene edges: ferroelectricity would not exist in the system for $K<1$. At the microscopic level, $K$ is a result of molecular dipole-graphene edge interactions as well as intricate intermolecular (dipoledipole) interactions. We note that, for polar molecules placed in front of a graphene sheet, the effect of the screening interaction (induced charge density in the monolayer) has been predicted to align their dipole normal to the bulk graphene basal plane, even at room temperature. ${ }^{29}$ However, the effective potential energy is more complex at the edge where it depends, e.g., on edge passivation. ${ }^{13}$ In consequence, the nontrivial interplay between the molecular adsorption at oxygen-passivated graphene edges (in the presence of an external electric field), graphene-molecule interactions, and molecule-molecule interactions determines both the equilibrium configuration of polar molecules at graphene edges (see examples in Supporting Information, Note 4) and the appearance of ferroelectricity in our devices.

Memcapacitive Behavior. Using eq 2, the experimental device capacitance $C\left(V_{\mathrm{g}}, p_{z}\right)=Q\left(V_{\mathrm{g}}, p_{z}\right) / V_{\mathrm{g}}$ is seen to be hysteretic, ambipolar, and divergent (Figure 5). These are

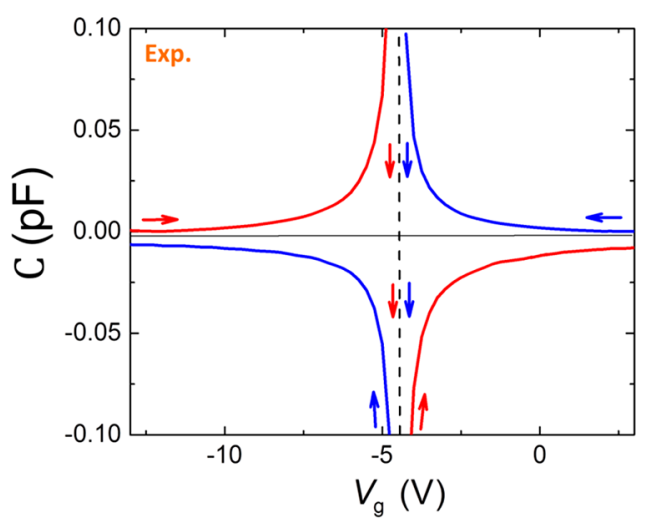

Figure 5. Memcapacitive behavior in graphene based transistors with polar molecules at edges for symmetric sweeps of $V_{g}$. Extracted ambipolar, hysteretic, and diverging capacitance in one of our devices (Device 1, Figure 1). This capacitance exhibits a typical behavior of predicted polarizable memcapacitive systems. ${ }^{5,6} V_{\text {CNP }}^{0}$ in the device is indicated by the vertical dashed line.

three unique features of predicted memory-capacitive systems. ${ }^{5,6}$ In our devices, this functionality arises at the molecular level as a result of the ferroelectricity of confined polar molecules at the graphene edges affecting the charge transport in the monolayer. The gate sweeps shown in Figure $2 \mathrm{~b}$ in effect demonstrate "write" and "read" operations for this memcapacitive device. At $t=0$, the $V_{\mathrm{CNP}}$ of the device is close to $V_{\mathrm{g}}=0 \mathrm{~V}\left(V_{\mathrm{CNP}}^{0}\right)$ since the polar molecules trapped at graphene edges are randomly distributed in the two available states $\left(p_{z}=0\right.$ since $\left.\eta=0\right)$. An increasing degree of polarization (corresponding to a "write" operation, gray line) occurs while increasing $V_{\mathrm{g}}$, with the stored state of the system observed as a change of the $V_{\mathrm{CNP}}$ to $\approx 20 \mathrm{~V}$ after returning to $V_{\mathrm{g}}=0 \mathrm{~V}$. Even after $30 \mathrm{~s}$ at $V_{\mathrm{g}}=0 \mathrm{~V}, V_{\mathrm{CNP}}$ remains at $\approx 20 \mathrm{~V}$ when performing subsequent "read" operations (red sweep, $0 \rightarrow$ $\left.V_{\mathrm{g}}^{\max }\right)$, and thus the state of this memcapacitive device is persistent at room temperature over unexpectedly long time scales given its molecular origin. 
In summary, we have demonstrated that polar molecules at the edges of an encapsulated graphene device display a twostate, switchable, and stable molecular dipole alignment in response to an external electric field, leading to a net dipole moment $p_{z}$, which causes a pronounced macroscopic charge bistability in gated graphene devices. This molecular system represents an experimental instance of envisioned memcapacitive devices ${ }^{5,6}$ and can be exploited for the exploration of correlated molecular systems ${ }^{4,24-26}$ and applications such as neuromorphic hardware architectures. ${ }^{7}$

\section{MATERIALS AND METHODS}

Device Fabrication. The fabrication process ${ }^{19}$ is based on the dry-pickup and encapsulation of graphene between hexagonal boron nitride $(\mathrm{hBN}) .{ }^{17,18}$ We select $\mathrm{hBN}$ flakes with thicknesses between 15 and $25 \mathrm{~nm}$ for our devices. Stacked hBN/graphene/hBN heterostructures are placed on top of a highly doped $\mathrm{Si}$ wafer with a $300 \mathrm{~nm} \mathrm{SiO}_{2}$ on top. Square devices are shaped from these heterostructures by electron beam lithography (EBL) and reactive ion etch (RIE) etching steps. The RIE process comprises an initial $\mathrm{SF}_{6}$ etch $(20 \mathrm{~s}$ ) for the top hBN (power $\approx 30 \mathrm{~W}, 30 \mathrm{sccm}$ ), a brief oxygen-based plasma etch (10 s) for graphene (power $\approx 40 \mathrm{~W}$, argon $40 \mathrm{sccm}$, oxygen $5 \mathrm{sccm})$ and a second $\mathrm{SF}_{6}$ etch $(20 \mathrm{~s})$ for the bottom etch (power $\approx 30 \mathrm{~W}, 30 \mathrm{sccm}$ ). We emphasize the usage of RIE etching as an effective way to reduce the edge disorder in graphene devices. ${ }^{22,30}$

The contacting procedure ${ }^{19}$ comprises a second EBL step performed to define the contacts of the device, followed by the evaporation of $\mathrm{Cr} / \mathrm{Pd} / \mathrm{Au}(2,15$, and $25 \mathrm{~nm}$, respectively) and a lift-off.

The definition of the device perimeter by etching leaves only the graphene edges exposed to the ambient environment. Here, the edge termination is essential in our devices. We expose the edge of graphene in our devices to both fluorinebased $\left(\mathrm{SF}_{6}\right)$ and oxygen $(\mathrm{O})$ plasmas, in order to evaluate the adsorption of polar molecules with respect to the edge passivation of graphene. Fluorine decoration at the edge is achieved by a plasma treatment at room temperature with $\mathrm{SF}_{6}$ as precursor gas. ${ }^{20,31}$ In other words, we have explicitly avoided the usage of $\mathrm{CF}_{4}$ plasma due to its tendency to introduce excessive disorder in graphene. ${ }^{20}$ Meanwhile, oxygen decoration at the edge is achieved by a short (1 s) plasma ashing (power $\approx 20 \mathrm{~W}$ ) post-treatment, a gentle, chemical, and undirected process. ${ }^{30}$

In practice, graphene is subjected to a fluorine edge treatment while etching the bottom $\mathrm{hBN}$ with the fluorinebased plasma (i.e., $\mathrm{SF}_{6}$ ), during the fabrication of $\mathrm{hBN}$ the encapsulated graphene devices. We note here that this may explain why the effect is previously unreported for encapsulated devices, where fluorine-based etching is used. ${ }^{17-19}$ Instead, oxygen decoration of graphene edges is achieved afterward by first annealing devices up to $400{ }^{\circ} \mathrm{C}$ in vacuum $\left(<10^{-5} \mathrm{mbar}\right)$ for $2 \mathrm{~h}$ to eliminate ${ }^{32}$ residual fluorine existent from the fabrication process, followed by exposure to $1 \mathrm{~s}$ of oxygen plasma to facilitate the complete oxidation of graphene edges. $^{30}$

Electrical Measurements. Our measurements were performed in a dark and environmentally controlled chamber. Measurements of resistance were performed using a Stanford SR830 lock-in amplifier with an excitation voltage of $200 \mu \mathrm{V}$ at a frequency of $17.77 \mathrm{~Hz}$. The gate-voltage sweeps were carried out at a rate of $0.2 \mathrm{~V} / \mathrm{s}$. We have confirmed the homogeneity ${ }^{33}$ of our fabricated devices by comparing the measurement in the configuration shown in Figure $1 \mathrm{~b}$, with a measurement carried out with the wiring configuration rotated by $90^{\circ} .{ }^{17,33} \mathrm{We}$ measure first devices where the edge of graphene is treated with $\mathrm{SF}_{6}$ as a result of the entire device fabrication (finished by etching the bottom hBN). We then measure devices after being subjected to the vacuum annealing and the $1 \mathrm{~s}$ oxygen plasma ashing processes, steps undertaken to remove fluorine and promote a complete oxygenation of the graphene edges in the device.

Atomistic Simulations. Our DFT calculations are performed with the software ATOMISTIX TOOLKIT. The wave functions are expanded in terms of a localized single- $\zeta$ polarized basis set and double- $\zeta$-polarized basis set. We use norm-conserving Troullier-Martins pseudopotentials with a density mesh cutoff of 75 hartrees, the LSDA exchangecorrelation by Perdew and Zunger ${ }^{34}$ and a $1 \times 5 \times 1(6 \times 6 \times 1) \mathrm{k}$ point grid with origin at $\Gamma$ for the ribbon calculations. We represent the direction perpendicular to the graphene plane as the $z$ direction (Figure 3a). In order to assess the robustness of the results with respect to intermolecular interactions, we introduce 1,2 , or 4 polar molecules per unit cell. Due to periodic boundary conditions in the $y$ direction, this corresponds to having an infinitely long edge with coverages $0.25,0.5$, and 1 of the bay sites. ${ }^{23}$ We place polar molecules with the center of mass slightly out of the graphene plane and then relax the structure. Geometry optimizations were performed until all forces were below $0.01 \mathrm{eV} / \AA$. Binding energies of all tested molecules adsorbed at the edge of graphene and equilibrium configurations are shown in the Supporting Information, Notes 3 and 4. We note that the optimized configurations with both single- and double- $\zeta$ polarized basis sets are in good agreement with other DFT studies in the literature at zero gate voltage. ${ }^{14,23}$

To simulate finite electrostatic gating, we add a charge to the graphene layer and solve the Poisson equation with Dirichlet (Neumann) boundary condition at the gate electrode (vacuum boundary). ${ }^{35}$

In our simplified model, the presence of the $\mathrm{hBN}$ dielectric and the possible presence of water molecules assembled at its edges are not explicitly accounted for; however, such effects would be included in the effective interaction parameter $K$. We do not expect dielectric screening effects to play a central role, as the experiments show that edge termination ( $\mathrm{O}$ or $\mathrm{F})$ is the main determinant for the amplitude of the hysteresis alongside the adsorbant molecular dipole moment.

We calculate the total energy $\varepsilon_{\text {tot }}$ of polar molecules adsorbed at the edge of a graphene nanoribbon passivated with fluorine or oxygen atoms, following experiments, and hydrogen, a common reference element used to passivate graphene edges. ${ }^{23}$

Although the simulated ribbon is much smaller than the experimental micrometer-size devices, the total energy difference $\varepsilon_{\text {tot }}^{(z<0)}-\varepsilon_{\text {tot }}^{(z>0)}$ is independent of the extent of the graphene basal plane. ${ }^{14}$ We have checked this by simulating ribbons of different widths $W(3,13$, and $23 \mathrm{~nm})$, obtaining very similar results in all these cases.

Furthermore, we have undertaken an appropriate electrostatic scaling by choosing the ribbon width $W$ and its distance to the gate electrode $b$ (Figure 3a). This is clearly seen in the generated carrier densities in the device, $n_{\text {avg }}$ (Figure $3 c$ ), having values similar to those observed in experiments. Thus, $E_{\text {loc }}$ and the electrostatic energy of interaction $\mu_{\mathrm{d}} E_{\text {loc }}$ (playing a 
role in $\varepsilon_{\text {tot }}$ ) are similar for simulated ribbons and experimental micrometer-size devices.

Finally, our DFT results show the same behavior for both zigzag and armchair termination of the graphene nanoribbon (Supporting Information, Note 5), providing a compelling reason for a robust charge bistability phenomenon to be present in experimental graphene devices, even with borders composed of a mixture of both edge chiralities.

\section{ASSOCIATED CONTENT}

\section{S Supporting Information}

The Supporting Information is available free of charge on the ACS Publications website at DOI: 10.1021/acs.nanolett.8b00797.

(1) Discussion of the measured effect in comparison with conventional charge hysteresis, (2) analysis of electrical field at the graphene edges, (3) atomistic calculations of binding energies of the adsorbed molecules, (4) symmetric positioning of different polar molecules at graphene edges, (5) DFT calculations on armchair edges, (6) statistical mechanics calculations regarding the molecular occupation at the edges, (7) electrostatic calculations of total charge in devices due to oriented dipoles, and (8) model for the molecular switching (PDF)

\section{AUTHOR INFORMATION}

\section{Corresponding Authors}

*E-mail: jcar@nanotech.dtu.dk.

*E-mail: peter.boggild@nanotech.dtu.dk.

*E-mail: mads.brandbyge@nanotech.dtu.dk.

\section{ORCID $\odot$}

Roman Sordan: 0000-0001-7373-0643

Peter Bøggild: 0000-0002-4342-0449

\section{Author Contributions}

J.M.C. conceived the idea and interpreted the data. J.M.C. and P.P. fabricated the devices and measured their electrical properties. G.C., A.I., T.G., and M.B. undertook the DFT simulations. J.M.C. and J.E.S. carried out the electrostatic calculation. M.B. developed the mean-field model. J.E.S. performed the statistical mechanics calculation. J.M.C., G.C., T.G., J.E.S., and M.B. carried out the data analysis. J.M.C., M.B., and P.B. wrote the manuscript, with comments from all authors.

\section{Funding}

This work was supported by the Danish National Research Foundation Center for Nanostructured Graphene, project DNRF103, the EU Seventh Framework Programme (FP7/ 2007-2013) under grant agreement number FP7-6040007 "GLADIATOR", and the EC Graphene FET Flagship, grant agreement number 604391. J.M.C. acknowledges funding from the Otto Mønsteds Fond. J.E.S. acknowledges support by the European Structural and Investment Funds in the FEDER component, through the Operational Competitiveness and Internationalization Program (COMPETE 2020) [Project no. 002797; Funding Reference: POCI-01-0247-FEDER-002797]

\section{Notes}

The authors declare no competing financial interest.

\section{ACKNOWLEDGMENTS}

We acknowledge stimulating discussions with A. Cagliani, M. Scardamaglia, and L. Camilli.

\section{REFERENCES}

(1) Jia, C.; Migliore, A.; Xin, N.; Huang, S.; Wang, J.; Yang, Q.; Wang, S.; Chen, H.; Wang, D.; Feng, B.; Liu, Z.; Zhang, G.; Qu, D.H.; Tian, H.; Ratner, M. A.; Xu, H. Q.; Nitzan, A.; Guo, X. Science 2016, 352 (6292), 1443-1445.

(2) Martínez-Blanco, J.; Nacci, C.; Erwin, S. C.; Kanisawa, K.; Locane, E.; Thomas, M.; von Oppen, F.; Brouwer, P. W.; Fölsch, S. Nat. Phys. 2015, 11 (8), 640-644.

(3) Zhang, Y.; Kersell, H.; Stefak, R.; Echeverria, J.; Iancu, V.; Perera, U. G. E.; Li, Y.; Deshpande, A.; Braun, K.-F.; Joachim, C.; Rapenne, G.; Hla, S.-W. Nat. Nanotechnol. 2016, 11 (8), 706-712.

(4) Sinha, S.; Santos, L. Phys. Rev. Lett. 2007, 99 (14), 140406.

(5) Krems, M.; Pershin, Y. V.; Di Ventra, M. Nano Lett. 2010, 10 (7), 2674-2678.

(6) Martinez-Rincon, J.; Di Ventra, M.; Pershin, Y. Phys. Rev. B: Condens. Matter Mater. Phys. 2010, 81 (19), 195430.

(7) Tran, D.; Teuscher, C. Int. J. Unconv. Comput. 2017, 13 (1), 3557.

(8) Schedin, F.; Geim, A. K.; Morozov, S. V.; Hill, E. W.; Blake, P.; Katsnelson, M. I.; Novoselov, K. S. Nat. Mater. 2007, 6, 652-655.

(9) Solís-Fernández, P.; Okada, S.; Sato, T.; Tsuji, M.; Ago, H. ACS Nano 2016, 10 (2), 2930-2939.

(10) Wehling, T. O.; Novoselov, K. S.; Morozov, S. V.; Vdovin, E. E.; Katsnelson, M. I.; Geim, A. K.; Lichtenstein, A. I. Nano Lett. 2008, 8 (1), 173-177.

(11) Chang, C.-H.; Fan, X.; Li, L.-J.; Kuo, J.-L. J. Phys. Chem. C 2012, 116 (25), 13788-13794.

(12) Yavari, F.; Kritzinger, C.; Gaire, C.; Song, L.; Gulapalli, H.; Borca-Tasciuc, T.; Ajayan, P. M.; Koratkar, N. Small 2010, 6 (22), $2535-2538$.

(13) Wang, W.; Li, Z. J. Appl. Phys. 2011, 109 (11), 114308.

(14) Berashevich, J.; Chakraborty, T. Phys. Rev. B: Condens. Matter Mater. Phys. 2010, 81 (20), 205431.

(15) Lin, X.; Ni, J.; Fang, C. J. Appl. Phys. 2013, 113 (3), 034306-6.

(16) Pak, A. J.; Paek, E.; Hwang, G. S. J. Phys. Chem. C 2014, 118 (38), 21770-21777.

(17) Wang, L.; Meric, I.; Huang, P. Y.; Gao, Q.; Gao, Y.; Tran, H.; Taniguchi, T.; Watanabe, K.; Campos, L. M.; Muller, D. A.; Guo, J.; Kim, P.; Hone, J.; Shepard, K. L.; Dean, C. R. Science 2013, 342 (6158), 614-617.

(18) Pizzocchero, F.; Gammelgaard, L.; Jessen, B. S.; Caridad, J. M.; Wang, L.; Hone, J.; Bøggild, P.; Booth, T. J. Nat. Commun. 2016, 7 , 11894.

(19) Pedrinazzi, P.; Caridad, J. M.; Mackenzie, D. M. A.; Pizzocchero, F.; Gammelgaard, L.; Jessen, B. S.; Sordan, R.; Booth, T. J.; Bøggild, P. Appl. Phys. Lett. 2018, 112 (3), 033101.

(20) Struzzi, C.; Sezen, H.; Amati, M.; Gregoratti, L.; Reckinger, M.; Colomer, J.-F.; Snyders, R.; Bittencourt, C.; Scardamaglia, M. Appl. Surf. Sci. 2017, 422, 104-110.

(21) Silvestrov, P. G.; Efetov, K. B. Phys. Rev. B: Condens. Matter Mater. Phys. 2008, 77 (15), 155436.

(22) Caridad, J. M.; Power, S. R.; Lotz, M. R.; Shylau, A. A.; Thomsen, J. D.; Gammelgaard, L.; Booth, T. J.; Jauho, A.-P.; Bøggild, P. Nat. Commun. 2018, 9 (1), 659.

(23) Abe, S.; Nagoya, Y.; Watari, F.; Tachikawa, H. Jpn. J. Appl. Phys. 2010, 49 (1), 01AH07.

(24) Fernández, M. S.; Peeters, F. M.; Neek-Amal, N. Phys. Rev. B: Condens. Matter Mater. Phys. 2016, 94 (4), 045436-5.

(25) Klinsmann, M.; Peter, D.; Büchler, H. P. New J. Phys. 2015, 17 (8), 085002.

(26) Gorshunov, B. P.; Torgashev, V. I.; Zhukova, E. S.; Thomas, V. G.; Belyanchikov, M. A.; Kadlec, C.; Kadlec, F.; Savinov, M.; Ostapchuk, T.; Petzelt, J.; Prokleska, J.; Tomas, P. V.; Pestrjakov, E. V.; Fursenko, D. A.; Shakurov, G. S.; Prokhorov, A. S.; Gorelik, V. S.; 
Kadyrov, L. S.; Uskov, V. V.; Kremer, R. K.; Dressel, M. Nat. Commun. 2016, 7, 12842.

(27) Griffiths, D. J. Introduction to electrodynamics; Prentice Hall, 1999.

(28) Gammelgaard, L.; Caridad, J. M.; Cagliani, A.; Mackenzie, D. M. A.; Petersen, D. H.; Booth, T. J.; Bøggild, P. 2D Mater. 2014, 1 (3), 035005

(29) Guinea, F.; Walet, N. R. Interaction between point charges, dipoles and graphene layers.https://arxiv.org/abs/1605.08429, 2016.

(30) Simonet, P.; Bischoff, D.; Moser, A.; Ihn, T.; Ensslin, K. J. Appl. Phys. 2015, 117 (18), 184303-05.

(31) Baraket, M.; Walton, S. G.; Lock, E. H.; Robinson, J. T.; Perkins, F. K. Appl. Phys. Lett. 2010, 96 (23), 231501.

(32) Ho, K.-I.; Huang, C.-H.; Liao, J.-H.; Zhang, W.; Li, L.-J.; Lai, C.-S.; Su, C.-Y. Sci. Rep. 2015, 4 (1), 5893.

(33) Mackenzie, D. M. A.; Buron, J. D.; Whelan, P. R.; Caridad, J. M.; Bjergfelt, M.; Luo, B.; Shivayogimath, A.; Smitshuysen, A. L.; Thomsen, J. D.; Booth, T. J.; Gammelgaard, L.; Zultak, J.; Jessen, B. S.; Bøggild, P.; Petersen, D. H. Nano Res. 2017, 10 (10), 3596-3605.

(34) Perdew, J. P.; Zunger, A. Phys. Rev. B: Condens. Matter Mater. Phys. 1981, 23 (10), 5048.

(35) Gunst, T.; Kaasbjerg, K.; Brandbyge, M. Phys. Rev. Lett. 2017, 118 (4), 046601. 